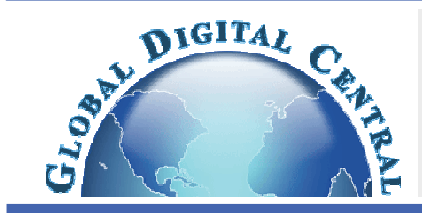

Frontiers in Heat and Mass Transfer

Available at www.ThermalFluidsCentral.org

\title{
NATURAL CONVECTIVE HEAT TRANSFER FROM A NARROW VERTICAL FLAT PLATE WITH A UNIFORM SURFACE HEAT FLUX AND WITH DIFFERENT PLATE EDGE CONDITIONS
}

\author{
Patrick H. Oosthuizen ${ }^{*}$, Jane T. Paul \\ Queen's University, Kingston, Ontario, K7L 3N6, Canada
}

\begin{abstract}
Natural convective heat transfer from narrow vertical plates which have a uniform surface heat flux has studied. With a narrow plate the heat transfer rate is dependent on the flow near the vertical edges of the plate. The magnitude of the edge effects will depend on the conditions existing near the edges of the plate. Three situations have here been considered these being a heated plate imbedded in a large plane adiabatic surface, the surfaces of the heated plane and the adiabatic surface being in the same plane, a heated plate with plane adiabatic surfaces above and below the heated plate, and a heated plate that is attached to a large parallel plane adiabatic surface but protrudes from the adiabatic plane surface. The effect of the edge condition on the heat transfer rate has here been numerically investigated.
\end{abstract}

Keywords: Natural Convection, Free Convection, Narrow Plates, Edge Effects, Numerical.

\section{INTRODUCTION}

Two-dimensional natural convective heat transfer from vertical isothermal plates has been extensively studied. However, when the width of the plate is relatively small compared to its height, the heat transfer rate can be considerably higher than that predicted by available two-dimensional flow results. The increase in the heat transfer rate from narrow plates relative to that from wide plates under the same conditions results from the fact that fluid flow is induced inwards near the vertical side edges of the plate and the flow near the edge of the plate is thus three-dimensional. This produces an increase in the heat transfer rate near the edges of the plate and this increase becomes significant when the plate is narrow, i.e., when its width is small compared to its height. The increase in the heat transfer rate when the plate is narrow is often said to be due to "three-dimensional effects" or "edge effects". Situations that can be approximately modeled as narrow vertical plates occur in a number of practical situations so a need exists to be able to predict heat transfer rates from such narrow plates. While there have been some limited studies of the natural convective heat transfer rate from narrow plates, the results obtained in these studies have been for a relatively narrow range of the governing parameters. There is still a need for a broader range of results that can be used as the basis for predicting heat transfer rates in practical situations. In the present study, the natural convective heat transfer rates from narrow vertical plates which have a uniform surface heat flux for a relatively

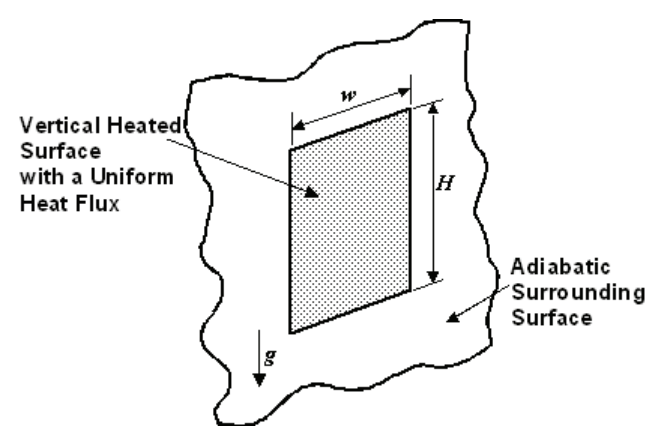

Fig. 1 Flow Situation considered when there are adiabatic side surfaces.

wide range of Rayleigh numbers and dimensionless plate widths have been numerically determined. Attention has been restricted to results for a Prandtl number of 0.7 , this being approximately the value existing in the application that originally motivated this study.

The magnitude of the edge effects will depend, in general, on the boundary conditions existing near the edge of the plate. To examine this effect, three situations have been considered. In one, the heated plate is imbedded in a large plane adiabatic surface, the surfaces of the heated plane and the adiabatic surface being in the same plane. In the second there are only plane adiabatic surfaces above and below the

\footnotetext{
* Patrick H. Oosthuizen.Email: oosthuiz@me.queensu.ca
} 


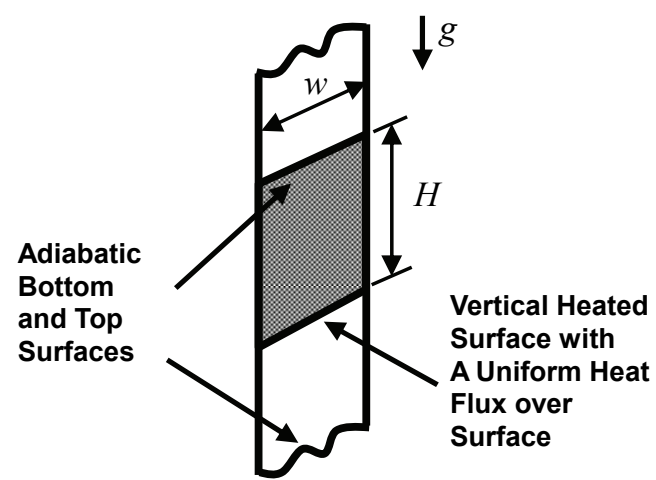

Fig. 2 Flow Situation considered when there are no side surfaces.

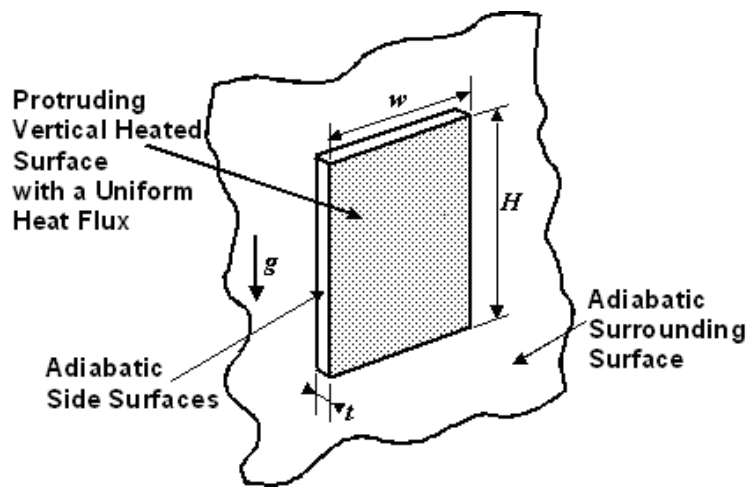

Fig. 3 Protruding Plate Flow situation considered.

heated plate and the edge of the plate is thus directly exposed to the surrounding fluid. The first two flow situations considered are thus as shown in Figs. 1 and 2. The width of the plate, $w$, is assumed to be of the same order of magnitude as the vertical height of the plate, $H$. In the second situation considered it is assumed that the plate and the adiabatic surfaces are thin. In addition to the situations shown in Figs. 1 and 2 attention has also be given to the case where the vertical heated plate is attached to a large parallel plane adiabatic surface and the heated plate protrudes by a small amount from the surrounding adiabatic plane surface on which it is mounted. There is again a uniform surface heat flux over the heated plate. The flow situation considered is thus as shown in Figs. 3 and 4 . Figure 3 shows the overall situation considered while Fig. 4 shows a vertical cross-section through the system. The "height" that the plate protrudes, $t$, has been assumed to be relatively small compared to the plate height, $H$. The value of $P_{t}=t / H$ in Fig. 4 is approximately 0.15 which is larger than the maximum value of $P_{t}$ for which results have here been obtained here.

Experimental studies of natural convective heat transfer from narrow vertical plates are described in Oosthuizen $(1965,1967)$. However, the conditions at the vertical edges of the plates used in these studies were very different from those assumed in the present study and the results obtained in the present study cannot therefore be quantitatively compared with those obtained in these experimental studies. A study of natural convective heat transfer from narrow vertical plates based on the use of the boundary layer equations is described in Oosthuizen and Paul (1985) and Oosthuizen and Henderson (1987). An experimental study of the free convection heat transfer rates from flush mounted and protruding microelectronic chips was undertaken by Park and Bergles (1985). In their study, the microelectronic chips were simulated by thin foil heaters. It was found that the Nusselt number increased as the chip width decreased. Even with the widest of their heaters, the heat transfer

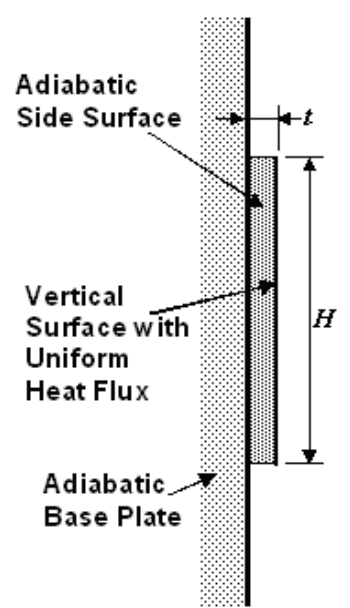

Fig. 4 Side view of protruding plate situation considered.

coefficients were higher than the values predicted by two-dimensional theory. Noto and Matsumoto $(1985,1987)$ and Churchill and Cho (1975) describe numerical studies of natural convective heat transfer from narrow vertical plates. However, a limited range of the governing parameters was covered in these studies making it difficult to draw general conclusions from the results. The present study is an extension of that described in Oosthuizen and Paul (2006, 2007a and 2007b). The effect of the conditions at the edge of the plate was not considered in any of these earlier studies. Some preliminary work on the effect of conditions at the edge of the plate was undertaken by Oosthuizen and Paul $(2007 c, 2007 d)$ and the present work is an extension of that described by Oosthuizen and Paul (2007d).

\section{SOLUTION PROCEDURE}

The flow has been assumed to be laminar and it has been assumed that the fluid properties are constant except for the density change with temperature which gives rise to the buoyancy forces, this having been treated by using the Boussinesq approach. It has also been assumed that the flow is symmetrical about the vertical centre-plane of the plate. The solution has been obtained by numerically solving the full threedimensional form of the governing equations, these equations being written in terms of dimensionless variables using the height, $H$, of the heated plate as the length scale and $q^{\prime} H / k$ as the temperature scale, $q$ ' being the uniform heat flux over the surface of the plate. Defining the following reference velocity:

$u_{r}=\frac{\alpha}{H} \sqrt{R a^{*} P r}$

where Pr is the Prandtl number and $\mathrm{Ra}^{*}$ is the heat flux Rayleigh number based on $\mathrm{H}$, i.e.:

$R a^{*}=\frac{\beta g q^{\prime} H^{4}}{k v \alpha}$

the following dimensionless variables have then been defined:

$$
\begin{gathered}
X=\frac{x}{H}, Y=\frac{y}{H}, Z=\frac{z}{H}, U_{X}=\frac{u_{x}}{u_{r}}, U_{Y}=\frac{u_{y}}{u_{r}}, \\
U_{Z}=\frac{u_{z}}{u_{r}}, P=\frac{\left(p-p_{F}\right) H}{\mu u_{r}}, \theta=\frac{T-T_{F}}{q^{\prime} H / k}
\end{gathered}
$$




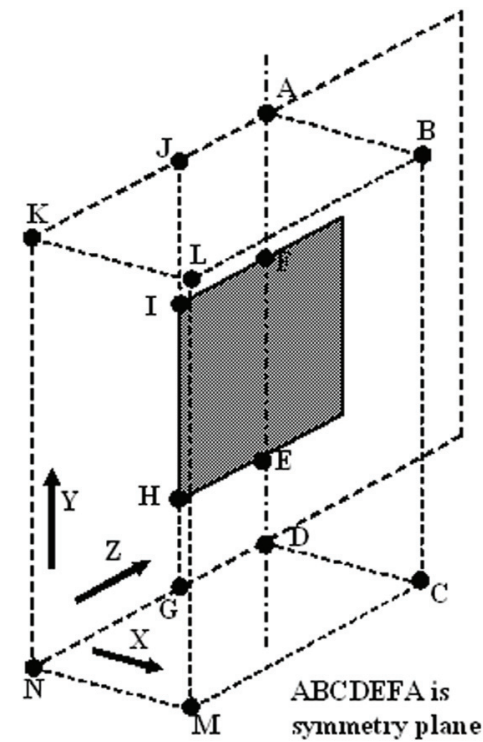

Fig. 5 Solution Domain ABCDIJLM

where $T$ is the temperature and $T_{F}$ is the fluid temperature far from the plate. The $X$ coordinate is measured in the horizontal direction normal to the plate, the $Y$-coordinate is measured in the vertically upward direction and the $Z$-coordinate is measured in the horizontal direction in the plane of the plate.

In terms of these dimensionless variables, the governing equations are:

$$
\frac{\partial U_{X}}{\partial X}+\frac{\partial U_{Y}}{\partial Y}+\frac{\partial U_{Z}}{\partial Z}=0
$$

$$
\begin{aligned}
U_{X} \frac{\partial U_{X}}{\partial X} & +U_{Y} \frac{\partial U_{X}}{\partial Y}+U_{Z} \frac{\partial U_{X}}{\partial Z} \\
& =\sqrt{\frac{P r}{R a^{*}}}\left(-\frac{\partial P}{\partial X}+\frac{\partial^{2} U_{X}}{\partial X^{2}}+\frac{\partial^{2} U_{X}}{\partial Y^{2}}+\frac{\partial^{2} U_{X}}{\partial Z^{2}}\right)
\end{aligned}
$$

$$
\begin{aligned}
U_{X} \frac{\partial U_{Y}}{\partial X} & +U_{Y} \frac{\partial U_{Y}}{\partial X}+U_{Z} \frac{\partial U_{Y}}{\partial X} \\
& =\sqrt{\frac{P r}{R a^{*}}}\left(-\frac{\partial P}{\partial Y}+\frac{\partial^{2} U_{Y}}{\partial X^{2}}+\frac{\partial^{2} U_{Y}}{\partial Y^{2}}+\frac{\partial^{2} U_{Y}}{\partial Z^{2}}\right)+\theta
\end{aligned}
$$

$$
\begin{aligned}
U_{X} \frac{\partial U_{Z}}{\partial X} & +U_{Y} \frac{\partial U_{Z}}{\partial Y}+U_{Z} \frac{\partial U_{Z}}{\partial Z} \\
& =\sqrt{\frac{P r}{R a}}\left(-\frac{\partial P}{\partial Z}+\frac{\partial^{2} U_{Z}}{\partial X^{2}}+\frac{\partial^{2} U_{Z}}{\partial Y^{2}}+\frac{\partial^{2} U_{Z}}{\partial Z^{2}}\right)
\end{aligned}
$$

$$
\begin{aligned}
U_{X} \frac{\partial \theta}{\partial X} & +U_{Y} \frac{\partial \theta}{\partial Y}+U_{Z} \frac{\partial \theta}{\partial Z} \\
& =\frac{1}{\sqrt{R a^{*} P r}}\left(\frac{\partial^{2} \theta}{\partial X^{2}}+\frac{\partial^{2} \theta}{\partial Y^{2}}+\frac{\partial^{2} \theta}{\partial Z^{2}}\right)
\end{aligned}
$$

Because the flow has been assumed to be symmetrical about the vertical center-line of the plate, the solution domain used in obtaining the solution is as shown in Fig. 5. ABCDEFA is the center-plane about which the flow is assumed to be symmetrical.
Considering the surfaces shown in Fig. 5, the assumed boundary conditions on the solution for the first edge condition considered are, in terms of the dimensionless variables since flow symmetry is being assumed:

FEHI: $\quad U_{X}=0, U_{Y}=0, U_{Z}=0, \frac{\partial \theta}{\partial X}=-1$

DNKA except for FEHI: $U_{X}=0, U_{Y}=0, U_{Z}=0, \frac{\partial \theta}{\partial X}=0$

BCML: $\quad U_{Y}=0, U_{Z}=0, \theta=0$

KLMN: $U_{X}=0, U_{Y}=0, \theta=0$

DCMN: $\quad U_{X}=0, U_{Z}=0, \theta=0$

ABCDEF: $U_{Z}=0, \frac{\partial U_{Y}}{\partial Z}=0, \frac{\partial U_{X}}{\partial Z}=0, \frac{\partial \theta}{\partial Z}=0$

For the second edge condition considered, since the heated plate and the adiabatic surfaces are assumed to be very thin and since both sides of the plate are assumed to have the same applied heat flux, the boundary conditions are assumed to be:

FEHI: $\quad U_{X}=0, U_{Y}=0, U_{Z}=0, \frac{\partial \theta}{\partial X}=-1$

DGJA except for FEHI: $U_{X}=0, U_{Y}=0, U_{Z}=0, \frac{\partial \theta}{\partial X}=0$

GNKJ: $\quad U_{X}=0, \frac{\partial U_{Y}}{\partial X}=0, \frac{\partial U_{Z}}{\partial X}=0, \frac{\partial \theta}{\partial X}=0$

BCML: $U_{Y}=0, U_{Z}=0, \theta=0$

KLMN: $\quad U_{X}=0, U_{Y}=0, \theta=0$

DCMN: $\quad U_{X}=0, U_{Z}=0, \theta=0$

ABCDEF: $U_{Z}=0, \frac{\partial U_{Y}}{\partial Z}=0, \frac{\partial U_{X}}{\partial Z}=0, \frac{\partial \theta}{\partial Z}=0$

The mean heat transfer rate from the heated plate has been expressed in terms of the following mean Nusselt number:

$N u=\frac{q^{\prime} H}{k\left(T_{H m}-T_{F}\right)}=\frac{1}{\theta_{H m}}$

where $T_{H m}$ and $\theta_{H m}$ are the mean temperature and the mean dimensionless temperature of the heated surface respectively.

The dimensionless governing equations subject to the boundary conditions discussed above have been numerically solved using the commercial finite-element solver, FIDAP. Extensive grid- and convergence criterion independence testing was undertaken. This indicated that the heat transfer results presented here are to within $1 \%$ independent of the number of grid points and of the convergencecriterion used. The effect of the positioning of the outer surfaces of the solution domain (i.e., surfaces MLBC, KLMN, DCMN, and ABLK in Fig. 5) from the heated surface was also examined and the positions used in obtaining the results discussed here were chosen to ensure that the heat transfer results were independent of this positioning to within one per cent.

The same basic procedure has been used to find the solution for the protruding plate case. The flow has again been assumed to be symmetrical about the vertical center-line of the plate and the flow has again been assumed to be laminar and it has been assumed that the fluid properties are constant except for the density change with temperature which gives rise to the buoyancy forces, this having again been treated by using the Boussinesq approach. The same set of dimensionless equations that were discussed above has been used to obtain the solution. 


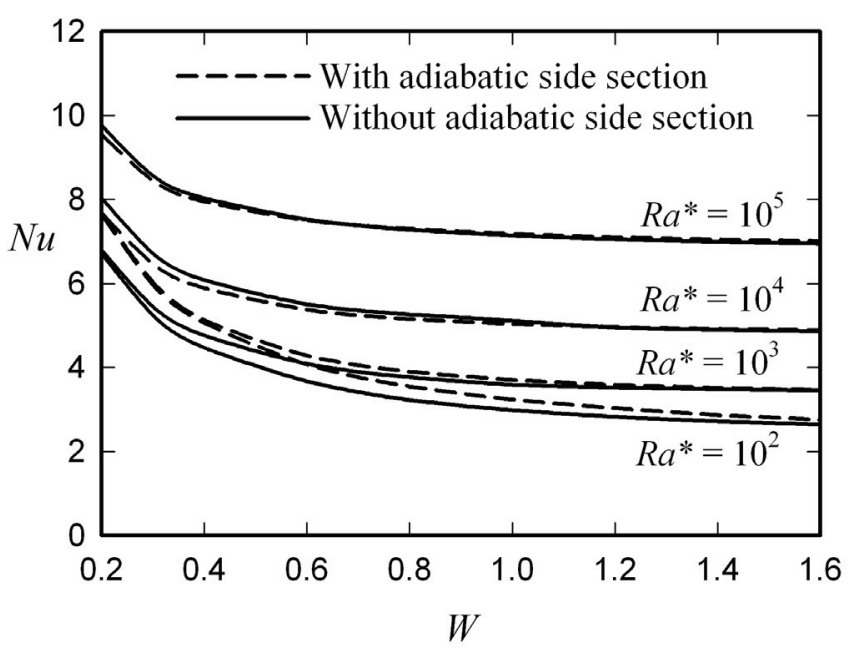

Fig. 6 Variation of mean Nusselt number with dimensionless plate width for various values of the heat flux Rayleigh number for the two edge conditions being considered.

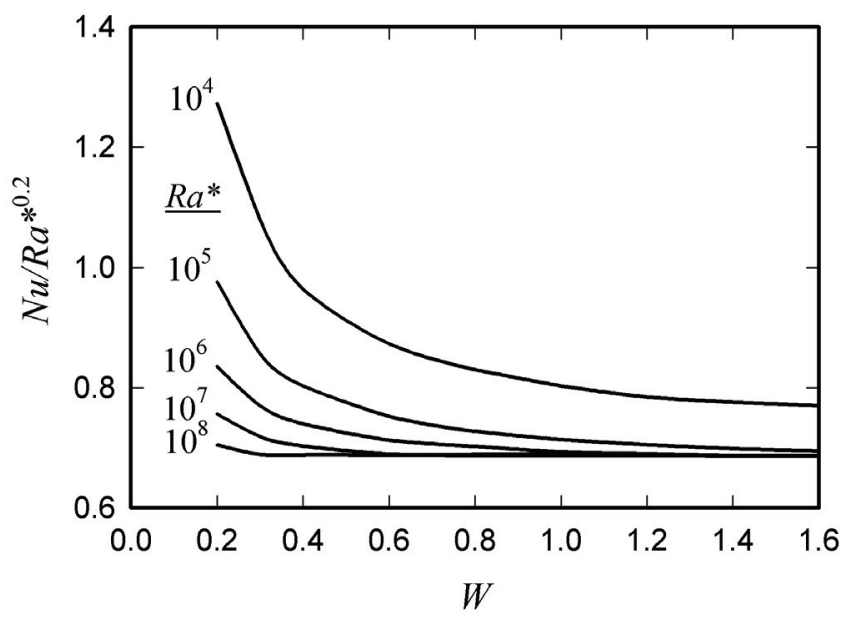

Fig. 7 Typical variations of $N u / R a^{* 0.2}$ with dimensionless plate width $W$ for the higher heat flux Rayleigh number values considered for the case where there are no side adiabatic sections.

\section{RESULTS}

The solution for the first two flow situations (those shown in Figs. 1 and 2) has the following parameters:

1. The heat flux Rayleigh number, $R a^{*}$, based on the plate height, $\mathrm{H}$, and the surface heat flux, $q$,

2. The dimensionless plate width, $W=w / H$

3. The Prandtl number, Pr.

4. The conditions at the edge of the plate

As already mentioned, results have only been obtained for $\mathrm{Pr}=$ 0.7. $\mathrm{Ra}^{*}$ values between 103 and 108 and $\mathrm{W}$ values between 0.2 and 1.6 have been considered.

Typical variations of the mean Nusselt number for the plate with dimensionless plate width for various heat flux Rayleigh numbers for the two side conditions being considered are shown in Fig. 6. It will be seen from these results that in all cases the mean Nusselt number tends to increase with decreasing $W$. It will further be noted that at the lower heat flux Rayleigh numbers considered, the Nusselt numbers for the case where there is no side section are lower than those for the case where there is a side section whereas at the higher heat flux Rayleigh

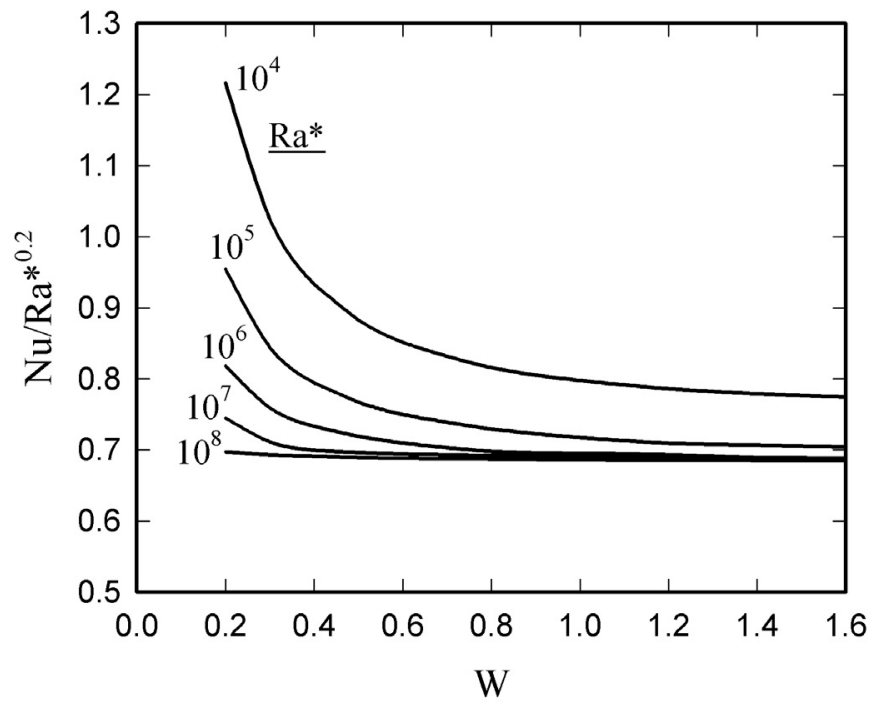

Fig. 8 Typical variations of $N u / R a^{* 0.2}$ with dimensionless plate width $W$ for the higher heat flux Rayleigh number values considered for the case where there are side adiabatic sections.

numbers the opposite is true. The rest of the discussion will focus on results for the higher heat flux Rayleigh numbers, i.e., for $R a^{*}$ values of $10^{4}$ and higher.

The effect of dimensionless plate width on the Nusselt number is further illustrated by the results shown in Figs. 7 and 8 for these higher heat flux Rayleigh numbers. In these figures it has been noted that with two-dimensional flow over a wide vertical plate that has a uniform heat flux over its surface:

$\frac{N u}{R a^{* 0.2}}=$ function $(P r)$

Figures 7 and 8 show the variations of $\mathrm{Nu} / \mathrm{Ra} * 0.2$ with the dimensionless plate width, $\mathrm{W}$, for various values of $\mathrm{Ra}^{*}$ for the case where there is no adiabatic side section (Fig. 7) and for the case where there is an adiabatic side section (Fig. 8). It will be seen that at the larger values of $\mathrm{W}$ and $\mathrm{Ra}^{*}$ the quantity $\mathrm{Nu} / \mathrm{Ra}^{*} 0.2$ does tend to a constant value of approximately 0.68 which is the value given by the similarity solution for two-dimensional flow over a wide plate with a uniform heat flux at the surface for a Prandtl number of 0.7 [Kakac and Yener (1995)]. It will also be seen from Figs. 7 and 8 that at the lowest values of $\mathrm{W}$ and $\mathrm{Ra}^{*}$ considered the value of $\mathrm{Nu} / \mathrm{Ra}^{*} 0.2$ is more than 80 per cent above the two-dimensional flow value. However, at the highest value of $\mathrm{Ra}^{*}$ considered the two-dimensional value applies at $\mathrm{W}$ values down to about 0.3 .

As mentioned before, the increase in the Nusselt number with decreasing $\mathrm{W}$ arises from the fact that there is an induced inflow towards the plate from the sides and this causes the heat transfer rate to be higher near the vertical sides of the plate than it is in the center region of the plate. This edge effect is illustrated by the results given in Figs. 9 and 10 which show the local dimensionless temperature distributions over the plate surface. The results given in these two figures illustrate how the edge effect grows more significant as $W$ is decreased and the extent of the edge regions thus increases compared to the overall plate area. These results also show how the extent of the edge region increases as $R a^{*}$ decreases, i.e., how the importance of the edge effect increases as the $R a^{*}$ decreases. Since the boundary layer thickness increases as $R a^{*}$ decreases this confirms that the extent of the edge region varies directly with the boundary layer thickness. 

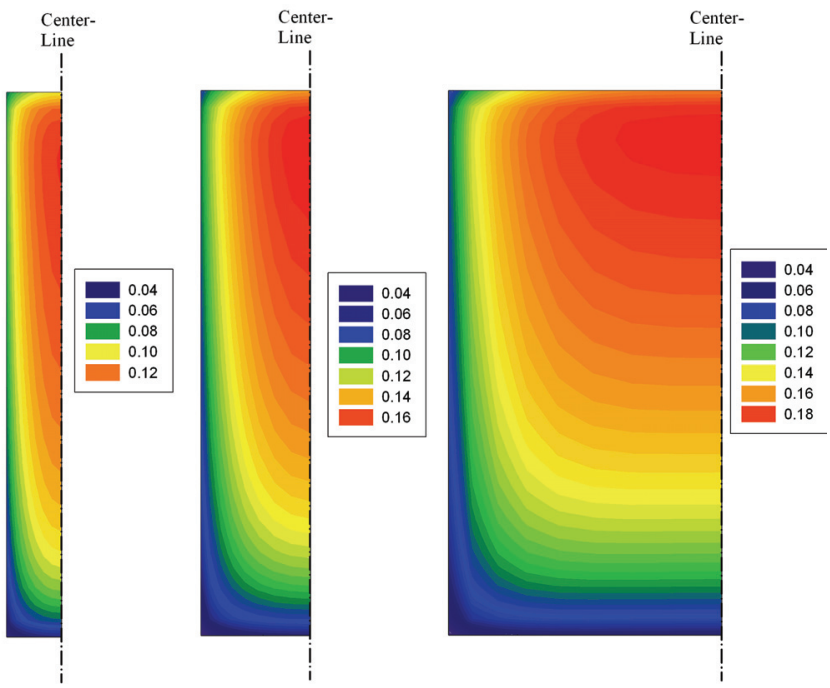

Fig. 9 Temperature distribution over surface of heated plate for $R a^{*}=$ $10^{5}$ and $W=0.2$ (left), 0.4 (center), and 1.0 (right).

In order to obtain an approximate equation for the Nusselt number for narrow plates, it has been assumed that for wide plates, i.e., for situations in which the edge effects are negligible, $\mathrm{Nu}$ is given for $\mathrm{Pr}=$ 0.7 , as mentioned above, by [10]:

$$
N u=0.68 R a^{* 0.2}
$$

As discussed above, Figs. 6, 7 and 8 show that this equation gives results that are in good agreement with the present numerical results at the larger values of $\mathrm{W}$ and $\mathrm{Ra}^{*}$ considered. Because of this assumed form of the variation of $\mathrm{Nu}$ with $\mathrm{Ra}^{*}$ when edge effects are negligible, it has been assumed that the Nusselt number for a plate of arbitrary dimensionless width $W$ is given by an equation of the form:

$\frac{N u}{R a^{* 0.2}}=0.68+$ function $\left(R a^{*}, W\right)$

The function of $R a^{*}$ and $W$ in this equation is assumed to depend on the ratio of the boundary layer thickness to the plate width, i.e., since the boundary layer thickness will depend on $H / R a^{* 0.2}$, it will depend on $1 / W R a^{* 0.2}$. The above equation has therefore been assumed to have the form:

$$
\frac{N u}{R a^{* 0.2}}=0.68+\text { function }\left(\frac{1}{W R a^{* 0.2}}\right)
$$

The form of the function was determined from the numerical results which indicated that the Nusselt number results obtained numerically could be approximately described for the case where there are side adiabatic sections by:

$\frac{N u}{R a^{* 0.2}}=0.68+\frac{0.75}{\left(W R a^{* 0.2}\right)^{1.4}}$

and for the case where there are no side adiabatic sections by:

$$
\frac{N u}{R a^{* 0.2}}=0.68+\frac{0.80}{\left(W R a^{* 0.2}\right)^{1.4}}
$$

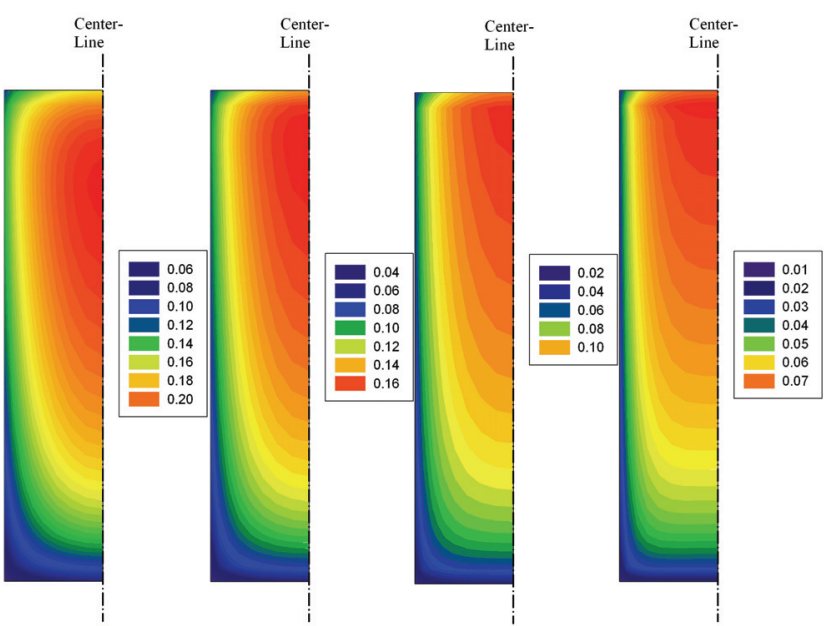

Fig. 10 Temperature distribution over surface of heated plate for $W=$ 0.4 and $R a^{*}=10^{4}$ (left), $10^{5}$ (middle left), $10^{6}$ (middle right), and $10^{7}$ (right).

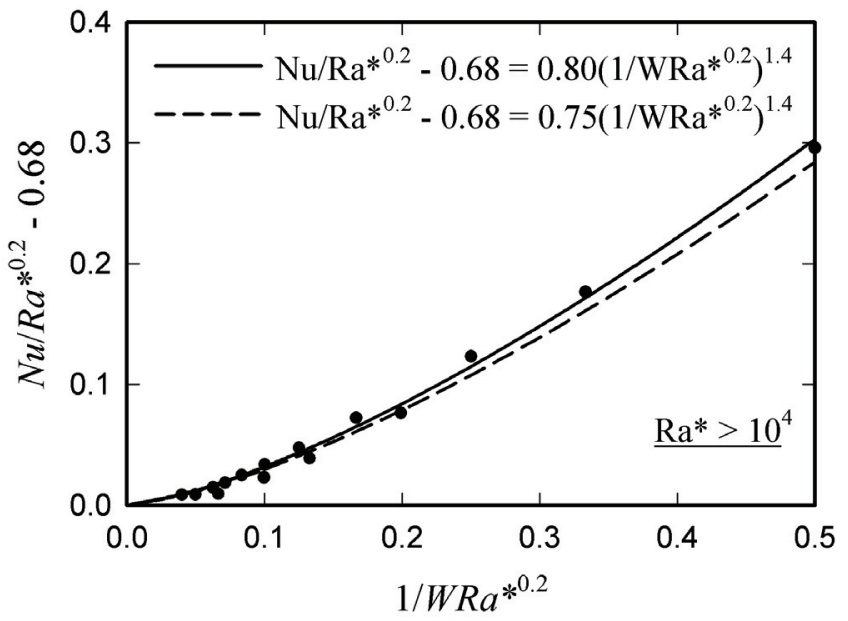

Fig. 11 Comparison of results given by Eq. (17) with numerical results for the no adiabatic side section case and with the results given by Eq. (16) for the case where there are adiabatic side sections.

This equation is compared with some of the numerical results for the case where there is no side adiabatic section in Fig. 11. The results given by Eq. (16) for the case where there are adiabatic side sections are also shown in this figure.

If three-dimensional effects (i.e., edge effects) are assumed to be negligible if:

$\frac{N u / R a^{* 0.2}-N u_{0} / R a^{* 0.2}}{N u_{0} / R a^{* 0.2}}=\frac{N u / R a^{* 0.2}-0.68}{0.68}<0.02$

i.e. if:

$\frac{N u}{R a^{* 0.2}}-0.68<0.014$

which gives, by using Eq. (16), for the case where there are side adiabatic sections: 


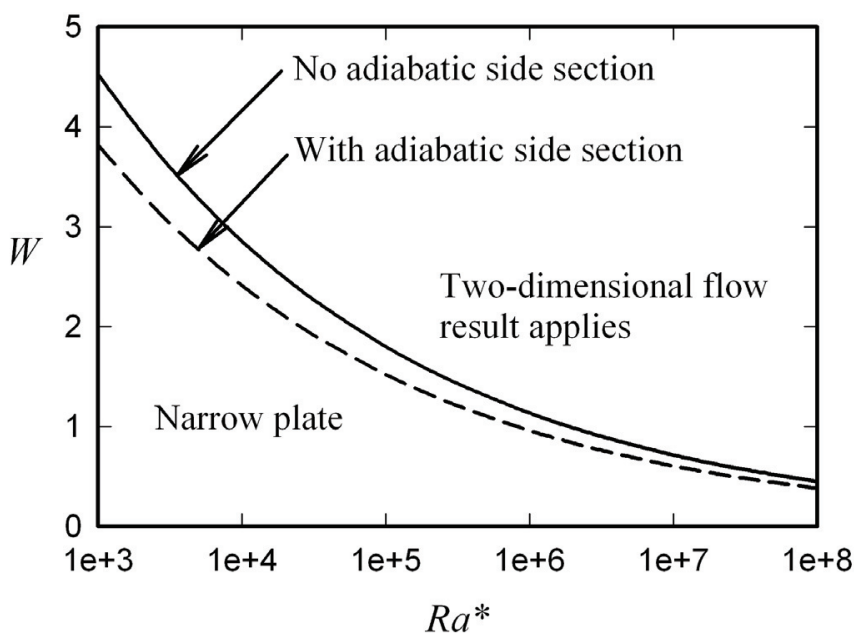

Fig. 12 Variation of $W$ value above which edge effects are negligible with $R a^{*}$ for the two edge conditions considered.

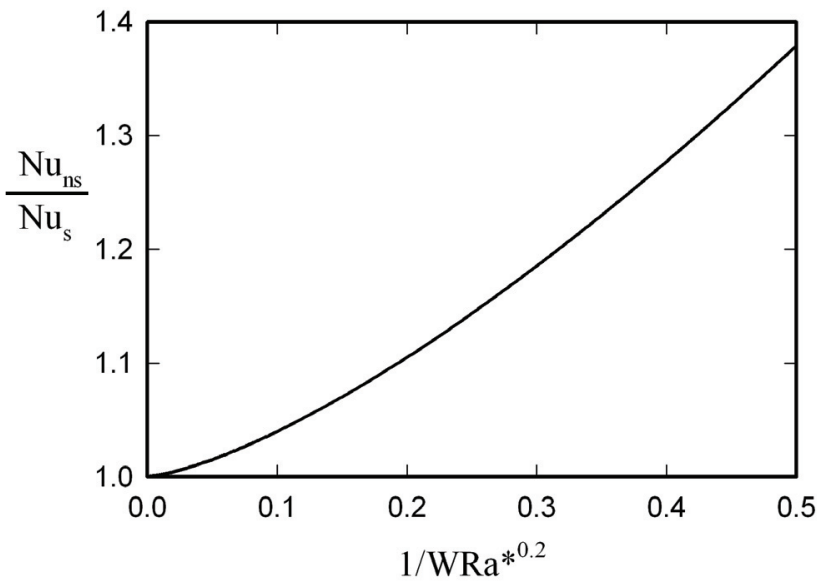

Fig. 13 Variation of ratio of Nusselt number for the no side section case to that for the case where there are side sections with $1 / W R a^{* 0.2}$ for $\mathrm{Ra}^{*}$ values of $10^{4}$ and higher.

$$
\begin{gathered}
\frac{0.75}{\left(W R a^{* 0.2}\right)^{1.4}}<0.014, \text { i.e. } W R a^{* 0.2}>15.2, \\
\text { i.e. } W>\frac{15.2}{R a^{* 0.2}}
\end{gathered}
$$

while for the case where there are no side sections, Eq. (17) gives:

$$
\begin{gathered}
\frac{0.80}{\left(W R a^{* 0.2}\right)^{1.4}}<0.014, \\
\text { i.e. } W R a^{* 0.2}>18.0, \\
\text { i.e., } W>\frac{18.0}{R a^{* 0.2}}
\end{gathered}
$$

Using these results, the variations of the values of $W$ above which three-dimensional effects on $\mathrm{Nu}$ are negligible (to within approximately $2 \%$ ) with $R a^{*}$ can be determined for the two cases being considered and are shown in Fig. 12.

It will also be noted that using Eqs. (16) and (17) gives the following approximate relation for the ratio of the Nusselt number for the no side adiabatic section case to that for the with-side adiabatic section case as:

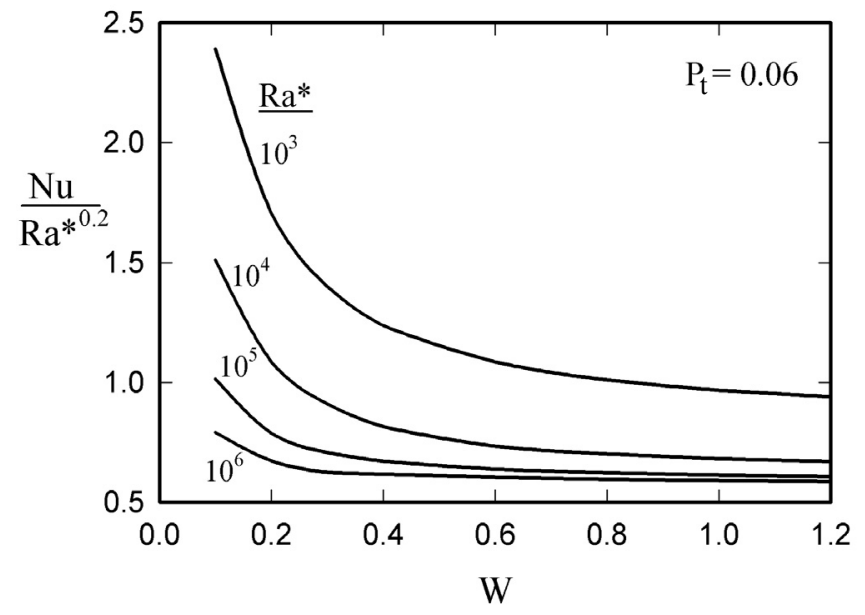

Fig. 14 Typical variation of $\mathrm{Nu} / \mathrm{R} a^{* 0.2}$ with dimensionless protruding plate width, $W$, for various values of $R a^{*}$. Results given are for a dimensionless protrusion distance, $P_{t}$, of 0.06 .

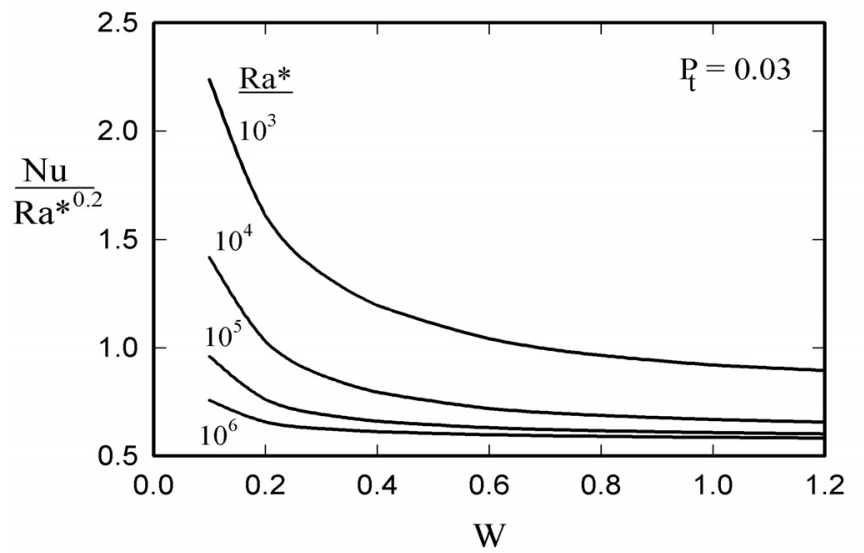

Fig. 15 Typical variation of $N u / R a^{* 0.2}$ with dimensionless plate width, $W$, for various values of $R a^{*}$. Results given are for a dimensionless protrusion distance, $P_{t}$, of 0.03 .

$\frac{N u_{n s}}{N u_{s}}=1+\frac{0.074}{\left(W R a^{* 0.2}\right)^{1.4}}$

The results given by this equation are shown in Fig. 13 .

Attention is next turned to the case of the protruding plate as shown in Figs. 2 and 3. The effect of plate width on the Nusselt number variation is illustrated by the results given in Figs. 14 and 15 which show typical variations of $N u / R a^{* 0.2}$ with dimensionless plate width, $W$ , for various heat flux Rayleigh numbers for the particular cases of $P_{t}=$ 0.06 and $P_{t}=0.03$. The heat transfer results are given in terms of $\mathrm{Nu} / \mathrm{Ra}^{* 0.2}$ because the classical analysis of heat transfer from a wide vertical plate with a uniform heat flux at the surface indicates, as noted above, that this quantity will be a constant for a given $P r$. It will be seen from these figures that as $W$ decreases the Nusselt number ratio increases, the increase for a particular value of $W$ increasing in relative magnitude with decreasing Rayleigh number. The increase is the result of edge effects, i.e., of the induced inward flow near the vertical side edges of the plate, this effect increasing in importance as $W$ and $R a^{*}$ decrease. The effect of $R a^{*}$ arises because the extent of the edge effect will depend on the boundary layer thickness relative to the plate width. The boundary layer thickness increases with decreasing $R a^{*}$ and the magnitude of the edge effect therefore increases with decreasing $R a^{*}$. 


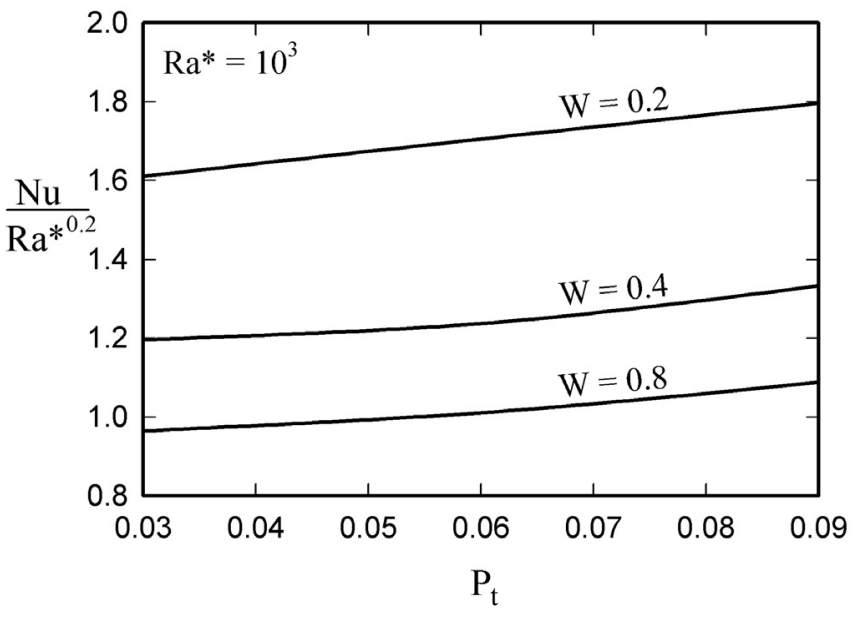

Fig. 16 Variation of $N u / R a^{* 0.2}$ with dimensionless protrusion distance, $P_{t}$, for various values of the dimensionless plate width, $W$, for $R a *=10^{3}$.

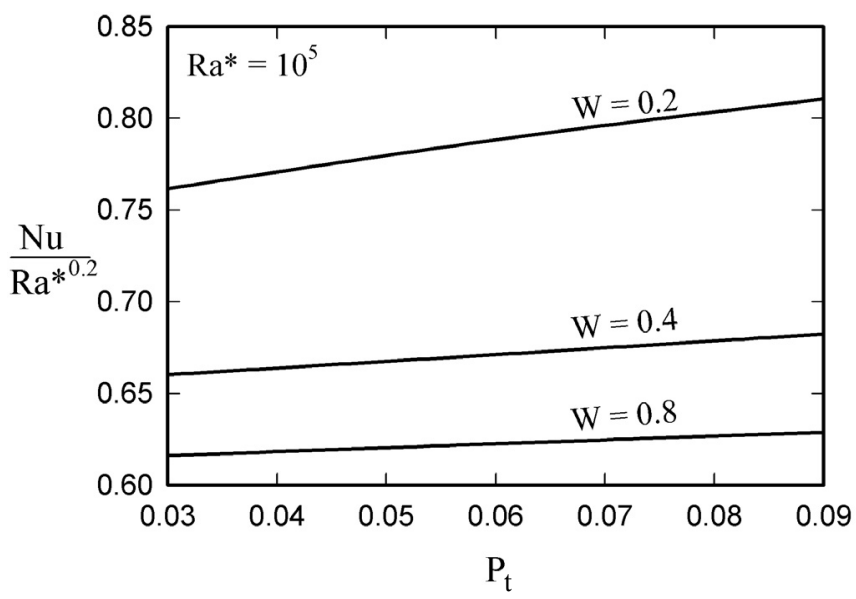

Fig. 17 Variation of $N u / R a^{* 0.2}$ with dimensionless protrusion distance, $P_{t}$, for various values of the dimensionless plate width, $W$, for $R a^{*}=10^{5}$.

The effect of the protrusion of the plate is further illustrated by the results given in Figs. 16 and 17 which show the variations of $\mathrm{Nu} / \mathrm{R} a^{* 0.2}$ with dimensionless protrusion "height", $P_{t}$, of the heated plate for various values of the dimensionless plate width and for Rayleigh numbers, $R a^{*}$, of $10^{3}$ (Fig. 16), and $10^{5}$ (Fig. 17). It will be seen from these figures that the Nusselt number ratio, $\mathrm{Nu} / \mathrm{Ra} a^{* 0.2}$, increase with increasing $P_{t}$, the effect increasing with decreasing $W$ and decreasing $R a^{*}$. It will also be seen from these figures that the percentage changes in the values of the Nusselt number ratio produced by changes in the value of $P_{t}$ are much less than the changes produced by changes in the value of $W$, the change produced by changing $P_{t}$ being of the order of $15 \%$ while the change produced by changing $W$ is of the order of $100 \%$.

The effect of the Rayleigh number on the Nusselt number ratio is more fully illustrated by the results given in Figs. 18 and 19 which show typical variations of $N u / R a^{* 0.2}$ with heat flux Rayleigh number for various dimensionless protrusion heights, $P_{t}$, for the particular cases of $W=1.2$ (Fig. 18) and $W=0.2$ (Fig. 19). It will be seen that the increase in the Nusselt number ratio, $N u / R a^{* 0.2}$, with decreasing $R a^{*}$ at the lower values of $R a^{*}$ and $W$ considered are much greater than the changes in the Nusselt number produced by changes in $P_{t}$.

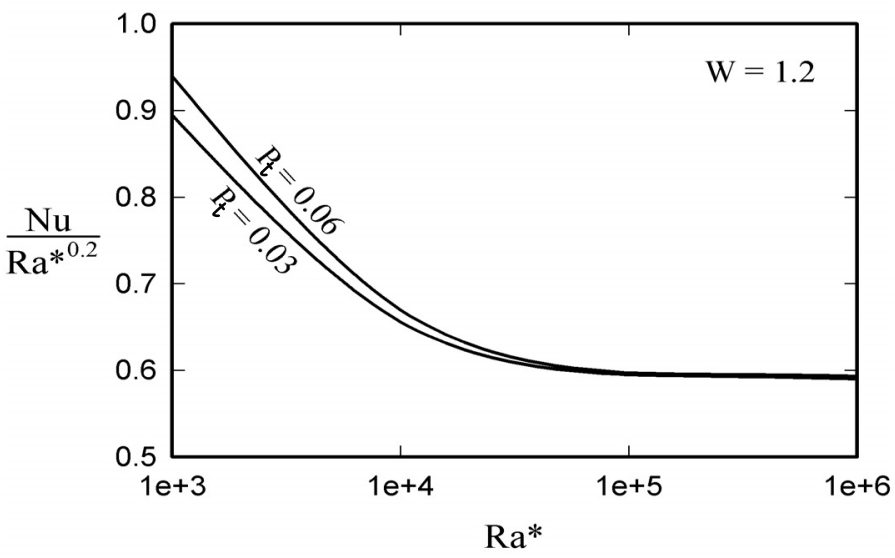

Fig. 18 Variation of $N u / R a^{* 0.2}$ with heat flux Rayleigh number, $R a^{*}$, for two values of the dimensionless protrusion distance, $P_{t}$, for $W=1.2$.

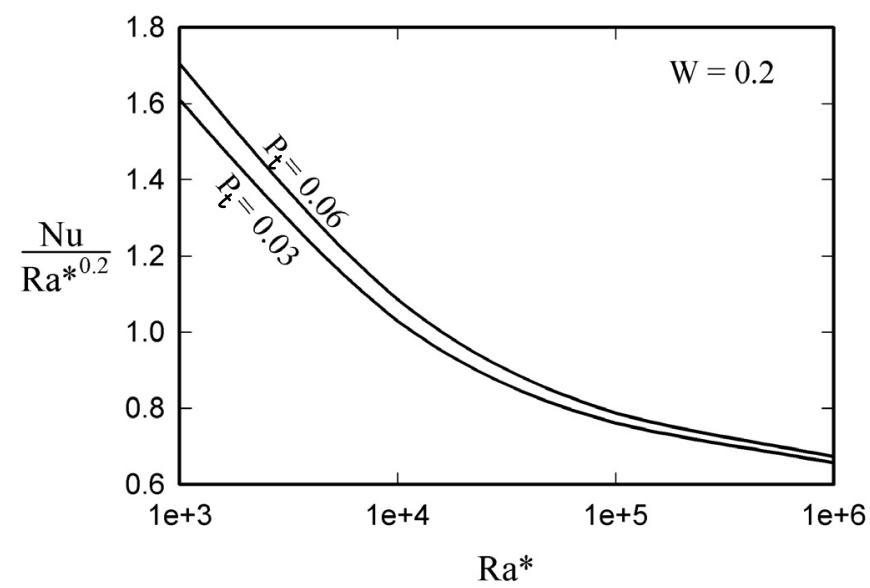

Fig. 19 Variation of $N u / R a^{* 0.2}$ with heat flux Rayleigh number, $R a^{*}$, for two values of the dimensionless protrusion distance, $P_{t}$, for $W=0.2$.

\section{CONCLUSIONS}

The dimensionless plate width has been shown to have a significant influence on the mean Nusselt number for natural convective heat transfer from a vertical flat plate with a uniform heat flux at the surface both for the case where there are side adiabatic sections and for the case where there are no adiabatic side sections. These edge effects increase with decreasing dimensionless plate width and decreasing Rayleigh number. Empirical equations for the mean heat transfer rate from narrow plates have been derived from the numerical results. These equations indicate that three-dimensional effects will be negligible for the case where there is a side-section if:

$$
W>\frac{15.2}{R a^{* 0.2}}
$$

and for the case where there is no side-section if:

$$
W>\frac{18.0}{R a^{* 0.2}}
$$

The protrusion of the heated plate from the surface tends to increase the Nusselt number, this effect increasing with decreasing heat flux Rayleigh number. 


\section{ACKNOWLEDGEMENTS}

This work was supported by the Natural Sciences and Engineering Research Council of Canada (NSERC).

\section{NOMENCLATURE}

$\begin{array}{ll}g & \text { gravitational acceleration }\left(\mathrm{m} / \mathrm{s}^{2}\right) \\ H & \text { height of heated plate }(\mathrm{m}) \\ k & \text { thermal conductivity of fluid }(\mathrm{W} / \mathrm{mK}) \\ N u & \text { mean Nusselt number based on } H \\ N u_{0} & \text { mean Nusselt number when edge effects are negligible } \\ N u_{s} & \text { mean Nusselt number for plate with side surfaces } \\ N u_{s n} & \text { mean Nusselt number for plate with no side surfaces } \\ P & \text { dimensionless pressure } \\ p & \text { pressure }(\mathrm{Pa}) \\ p_{F} & \text { pressure in fluid }(\mathrm{Pa}) \\ P r & \text { Prandtl number } \\ P_{t} & \text { dimensionless distance that plate protrudes, } t / H \\ q^{\prime} & \text { heat flux at surface of plate }\left(\mathrm{W} / \mathrm{m}^{2}\right) \\ R a^{*} & \text { heat flux Rayleigh number based on } H \\ T & \text { temperature }(\mathrm{K}) \\ T_{F} & \text { temperature of fluid }(\mathrm{K}) \\ T_{H m} & \text { mean plate temperature }(\mathrm{K}) \\ t & \text { distance that plate protrudes }(\mathrm{m}) \\ U_{X} & \text { dimensionless velocity component in } X \text { direction } \\ u_{x} & \text { velocity component in } x \text { direction }(\mathrm{m} / \mathrm{s}) \\ u_{r} & \text { reference velocity }(\mathrm{m} / \mathrm{s}) \\ U_{Y} & \text { dimensionless velocity component in } Y \text { direction } \\ u_{y} & \text { velocity component in } y \text { direction }(\mathrm{m} / \mathrm{s}) \\ U_{Z} & \text { dimensionless velocity component in } Z \text { direction } \\ u_{z} & \text { velocity component in } z \text { direction }(\mathrm{m} / \mathrm{s}) \\ w & \text { width of plate }(\mathrm{m}) \\ W & \text { dimensionless width of plate, } w / H \\ X & \text { dimensionless horizontal coordinate normal to plate } \\ x & \text { horizontal coordinate normal to plate }(\mathrm{m}) \\ Y & \text { dimensionless vertical coordinate } \\ \theta_{H m} & \text { vertical coordinate }(\mathrm{m}) \\ v & \text { dimensionless horizontal coordinate in plane of plate } \\ Z & \text { horizontal coordinate in plane of plate }(\mathrm{m}) \\ z & \text { dimensionless mean plate temperature } \\ G r e e k ~ S y m b o l s \\ \alpha & \text { bulk expansion coefficient } \mathrm{K}^{-1} \\ & \text { dimensionless temperature } \\ & \end{array}$

\section{REFERENCES}

Churchill, S.W., and Chu, H.H.S., 1975, "Correlating Equations for Laminar and Turbulent Free Convection from a Vertical Plate," International Journal of Heat and Mass Transfer, 18, 1323-1329. DOI:10.1016/0017-9310(75)90243-4

Kakac, S., and Yener, Y., 1995, Convective Heat Transfer, $2^{\text {nd }}$ ed., CRC Press, Boca Raton, FL, pp. 320-322.

Noto, K., Matsumoto, R. 1985, "Three-dimensional Analysis of the Navier-Stokes on Laminar Natural Convection Around a Vertical Flat Plate", Numerical Methods in Laminar and Turbulent Flow Proceedings of the Fourth International Conference, University College of Swansea, Wales, 4(1), pp. 865-877.
Noto, K., Matsumoto, R., 1987, "Three-dimensional Numerical of Natural Convective Heat Transfer from a Vertical Flat Plate", Proceedings of the 1987 American Society of Mechanical EngineersJapan Society of Mechanical Engineering Thermal Engineering Joint Conference, Vol. 5, pp. 1-8, Honolulu.

Oosthuizen, P.H., 1965, "An Experimental Analysis of the Heat Transfer by Laminar Free Convection from a Narrow Vertical Plate," Journal, South African Institute of Mechanical Engineering, 14(7), 153-158.

Oosthuizen, P.H., 1967, "A Further Experimental Study of the Laminar Free Convective Heat Transfer from Narrow Vertical Plates in Air," Journal, South African Institute of Mechanical Engineering, 16(9), 182-184.

Oosthuizen, P.H., and Paul, J.T., 1985, "Numerical Study of Free Convective Heat Transfer from Narrow Vertical Flat Plates," Proceedings of the $10^{\text {th }}$ Canadian Congress of Applied Mechanics, pp. C23-C24, London, ON.

Oosthuizen, P.H., and Henderson, C., 1987, "Edge Effects on Forced and Free Convective Laminar Boundary Flow Over a Flat Plate," Proceedings of the 1987 American Society of Mechanical Engineers, Winter Annual Meeting, HTD-Vol. 82 Convective Transport, pp. 149155, Anaheim, CA.

Oosthuizen, P.H., and Paul, J.T., 2006, "Natural Convective Heat Transfer from a Narrow Isothermal Vertical Flat Plate," AIAA 2006 3397, Proceedings of the $9^{\text {th }}$ American Institute of Aeronautics and Astronautics / American Society of Mechanical Engineers Joint Thermophysics \& Heat Transfer Conference, pp. 1-8, San Francisco, CA.

Oosthuizen, P.H., and Paul, J.T., 2007a, "Natural Convective Heat Transfer from a Narrow Vertical Flat Plate with a Uniform Heat Flux at the Surface," HT2007-32134, Proceedings of the American Society of Mechanical Engineers-Japan Society of Mechanical Engineering Thermal Engineering Summer Heat Transfer Conference, Vancouver, BC.

Oosthuizen, P.H. and Paul, J.T., 2007b, "Natural Convective Heat Transfer from a Recessed Narrow Vertical Flat Plate with a Uniform Heat Flux at the Surface," Proceedings of the $5^{\text {th }}$ International Conference on Heat Transfer, Fluid Mechanics \& Thermodynamics HEFAT2007, Sun City, RSA.

Oosthuizen, P.H. and Paul, J.T., 2007c, "Natural Convective Heat Transfer from a Narrow Vertical Isothermal Flat Plate with Different Edge Conditions," Proceedings of the $15^{\text {th }}$ Annual Conference of the Computational Fluid Dynamics Society of Canada, Toronto, ON.

Oosthuizen, P.H. and Paul, J.T., 2007d, "Effect of Edge Conditions on Natural Convective Heat Transfer from a Narrow Vertical Flat Plate with a Uniform Surface Heat Flux," IMECE2007-42712, Proceedings of the American Society of Mechanical Engineers International Mechanical Engineering Congress and Exposition.

Park, K.A., and Bergles, A.E., 1985, "Natural Convection Heat Transfer Characteristics of Simulated Microelectronic Chips," Heat Transfer in Electronic Equipment 1985, Proceedings of the $23^{\text {rd }}$ American Society of Mechanical Engineers National Heat Transfer Conference, pp. 2937, Denver 